\title{
The method and mathematical support for calibration of a goniometric system based on the accelerometers
}

\author{
$A V$ Grecheneva $^{1}, N V$ Dorofeev $^{1}, O R$ Kuzichkin $^{1, *}, R V$ Romanov $^{2}$, and $D I$ Surzhik $^{2}$ \\ ${ }^{1}$ Belgorod State University, 85 Pobedy St, Belgorod, 308015, Russia \\ ${ }^{2}$ Vladimir State University, 87 Gor'kogo St, Vladimir, 600000, Russia
}

\begin{abstract}
The article is devoted to the development of a calibration method for the goniometric system, which includes accelerometers. The complexity of the technological process of mechanical calibration of accelerometers was noted. It is proposed to apply software-algorithmic correction of measurement results. A mathematical model of signals of an accelerometer converter is described, which takes into account the influence of measurement errors. It is noted that in order to find the parameters of the mathematical model of an accelerometer signal, it is necessary to perform calibration. A mathematical model and the principle of calibration of the misalignment angle of the axes of the accelerometer are proposed and described. To evaluate the effectiveness of the proposed calibration method, experimental studies and data processing of the accelerometer were carried. As a result of experimental studies, an estimation of the degree of error compensation of the ADIS 16534 accelerometer measurements was performed with the application of the modified multi-position calibration method and the calibration method proposed by the authors of this article.
\end{abstract}

\section{Introduction}

Currently, the use of modern microprocessor signal processing devices and high accuracy of microelectromechanical (MEMS) sensors allow the development of precision measurement systems.

Since the MEMS accelerometer is an electromechanical device, after initial adjustment and installation in the goniometric control system, a static "load" continues to operate on the device. This causes a change in the sensitivity of the measurements and the displacement of the zero level of the goniometer. As a result, it's leads to an increase in the measurement error relative to the specified error [1-2]. Therefore, it is necessary to perform additional calibration of the misalignment angle and the accelerometer measurement sensitivity.

The application of the method of mechanical calibration to strict parallelism of the base axes is a complex and time-consuming technological process [3]. When performing

\footnotetext{
* Corresponding author: 1155464@bsu.edu.ru
} 
calibration operations, special technical means are used to set and control certain spatial positions of the housing of the accelerometer [4]. The most convenient and expedient is the path of program-algorithmic correction of the results of measurements of acceleration projections, i.e. output signals of accelerometers, based on mathematical models that take into account possible angular deviations of the base axes. Therefore, the stage of theoretical studies, including mathematical modeling and analysis of instrumental errors, is an important stage both in solving the problem of increasing the accuracy of measurements, and developing a common technological process for designing and creating angular measurement systems [5].

The aim of this work is to develop of a simple method for calibrating accelerometers by means of algorithms for processing a small set of measurements under the action of gravity acceleration.

\section{The mathematical model of the accelerometer signals}

Given the initial displacement and sensitivity of the accelerometer, its signals can be represented as:

$$
\left\{\begin{array}{l}
a_{x}=a_{0}+k_{a x} \cdot a^{x}{ }_{\text {real }} \cdot \cos (\Delta \alpha) ; \\
a_{y}=a_{0}+k_{a y} \cdot a^{y}{ }_{\text {real }} \cdot \sin (\Delta \beta) ;
\end{array}\right.
$$

where, $a_{x}, a_{y}$ are the output values of the accelerometer; $a^{x}{ }_{\text {real }}, a^{y}$ real are the actual values of the acceleration acting on the sensor; $a_{0}$ is the initial offset; $k_{a x}, k_{a y}$ are the sensitivity coefficients; $\Delta \alpha, \Delta \beta$ are the angles between the direction of the acceleration vector $\vec{a}$ by the axes of the sensitivity of the accelerometer.

When considering the work of the accelerometric goniometer, the construction of which is described in the works of the authors of this article [6,7], the error in determining the angle [8] can be estimated using the equation:

$$
I(t)=a(t)+\Delta \varphi+S F \cdot a(t)+N \cdot a(t)+\varepsilon(t)
$$

where, $I(t)$ are signals from sensor; $a(t)$ is the acceleration vector; $\Delta \varphi$ is the offset of zeros (the systematic error); $S F$ is the matrix scaling factors; $N$ is the matrix of nonorthogonality of accelerometer axes in relation to the $\mathrm{X}$ axis; $\varepsilon(t)$ are the noise of the transducers.

The synthesis of the data processing algorithm for the goniometric control system assumes knowing of the parameters of the mathematical model (2). For this purpose, the calibration procedure is performed.

\section{The mathematical support for the calibration of the goniometric system}

The main purpose of conducting calibration operations is to determine the numerical values of the constants inherent in a specific design of the sensor part. These operations are presented as the result of separate stages of experimental research with the initial definition of work efficiency of the sensor. Suppose that the accelerometer system for measuring the angle is not balanced, i.e. there is the error of the angle of misalignment:

$$
\varphi=\pi / 2-\Delta \varphi
$$


where $\Delta \varphi$ is the angle of misalignment.

$$
K_{x}=K_{y} \cdot(\Delta K+1),
$$

where $\Delta K$ is the coefficient of the orthogonal mismatch of the accelerometer axes.

According to the proposed method, compensation of the angle of misalignment is carried out when registering acceleration values at the moment of fixation of the accelerometer and after rotating the coordinate system along the orthogonal direction by $90^{\circ}$ (Figure 1).

In this case, the coefficient of the orthogonal mismatch will be determined from the equation:

$$
\Delta K=\left(U_{x} / U_{x}^{\prime}\right)-1
$$

When the OX accelerometer axis is rotated on the $\pi / 2$ (Figure 2) in the counterclockwise direction, the acceleration signals will take the form:

$$
\left\{\begin{array}{c}
U_{x_{1}}=G \cdot \cos \alpha ; \\
U_{y_{1}}=G \cdot \sin (\Delta \varphi+\alpha) ; \\
U_{x_{2}}=G \cdot \sin \alpha ; \\
U_{y_{2}}=-G \cdot \cos (\Delta \varphi+\alpha) .
\end{array}\right.
$$
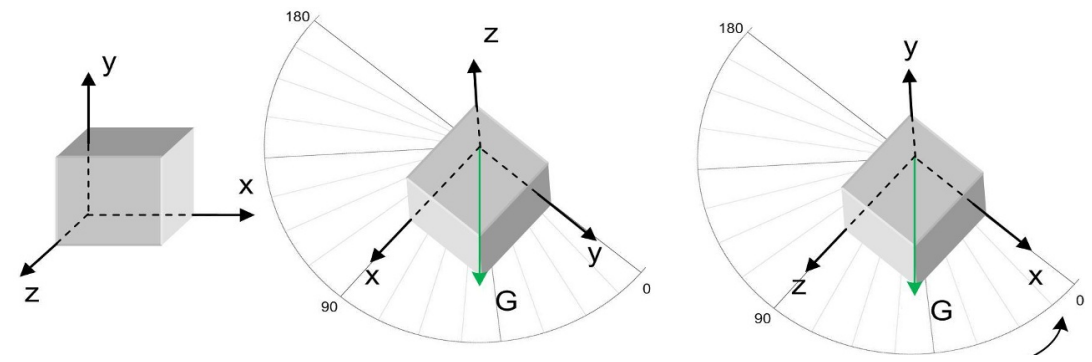

Fig. 1. Orientation of the accelerometer axes relative to the plane of the local coordinate system for the angle of misalignment calibration. a) Accelerometer coordinate system; b) Recording of accelerations in the accelerometer initial position; c) Registration of accelerations during rotation of the coordinate system.

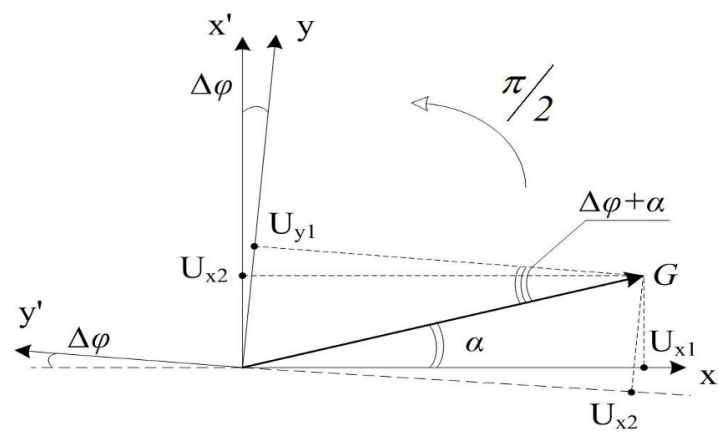

Fig. 2. The scheme for determining of the angle of misalignment.

To determine the angle of misalignment, the following notation must be entered:

$$
\begin{aligned}
& a=U_{y_{1}} / U_{x_{1}}=\sin (\Delta \varphi+\alpha) / \cos \alpha=\sin \Delta \varphi+\cos \Delta \varphi \cdot \operatorname{tg} \alpha ; \\
& b=U_{y_{2}} / U_{x_{2}}=-\cos (\Delta \varphi+\alpha) / \sin \alpha=-\cos \Delta \varphi \cdot \operatorname{ctg} \alpha+\sin \Delta \varphi .
\end{aligned}
$$

Solving equation (7), the angle $\alpha$ can be determined by the formula: 


$$
\operatorname{tg} \alpha=\frac{a-\sin \Delta \varphi}{\cos \Delta \varphi} \Rightarrow \operatorname{ctg} \alpha=\frac{\cos \Delta \varphi}{a-\sin \Delta \varphi} .
$$

When substituting of the equation (9) the equation (10) takes the form:

$$
\begin{gathered}
b=-\cos \Delta \varphi \cdot \frac{\cos \Delta \varphi}{\alpha-\sin \Delta \varphi}+\sin \Delta \varphi-\cos ^{2} \Delta \varphi=(b-\sin \Delta \varphi)(a-\sin \Delta \varphi)= \\
=a \cdot b+\sin ^{2} \Delta \varphi-(a+b) \sin \Delta \varphi .
\end{gathered}
$$

As a result of mathematical operations, equation (10) takes the form:

$$
1+a \cdot b=(a+b) \sin \Delta \varphi
$$

Further, taking into account the condition, the determination of the angle of misalignment of the accelerometer axes is possible by the formula:

$$
\sin \Delta \varphi=\frac{1+a \cdot b}{a+b} \Rightarrow \Delta \varphi=\arcsin \left(\frac{1+a \cdot b}{a+b}\right)
$$

It should be noted that the compensation of the error of the non-orthogonality of the axes of the sensor element in the accelerometer to the sensor microchip is due to the application of the automatic positioning method. The main provisions of this method were developed by the authors of this article and have already been described earlier in [9]. According to this method, the angle of rotation of the coordinate system of the accelerometer is correlated with the idealized coordinate system.

\section{Results and conclusions}

To evaluate the effectiveness of the proposed method, experimental studies and data processing of the accelerometer were carried out based on two methods of calibration: the proposed in this article and the modified multi-position method (MMPM) [10]. According to the MMPM, measurements are made at a minimum of 9 positions of a sensor with a known angular orientation, and the parameter estimates are obtained by the method of least squares. The disadvantage is the need to specify the known and high precision angular orientation of the accelerometer axes relative to the axes of the local coordinate system. The calibration procedure described in this article is devoid of this lack.

The results of experimental studies, reflected in Table 1, allow estimating the degree of errors compensation in measuring the accelerometer ADIS16534 with the use of the standard calibration method and the calibration method proposed by the authors of this article. In addition, data from the sensor specification are also given [11].

Table 1. The results of experimental studies of ADIS16534.

\begin{tabular}{|c|c|c|c|c|c|c|c|c|}
\hline & \multicolumn{2}{|c|}{$\begin{array}{c}\text { The average value } \\
\text { of measurement } \\
\text { results }\end{array}$} & \multicolumn{2}{|c|}{$\begin{array}{c}\text { The average value of } \\
\text { calibration results by } \\
\text { the proposed method }\end{array}$} & $\begin{array}{c}\text { The average value of } \\
\text { the calibration results } \\
\text { of the MWRM }\end{array}$ & \multicolumn{2}{|c|}{$\begin{array}{c}\text { Data from the } \\
\text { specification }\end{array}$} \\
\hline Parameter/ axis & $\mathrm{X}$ & $\mathrm{Y}$ & $\mathrm{X}$ & $\mathrm{Y}$ & $\mathrm{X}$ & $\mathrm{Y}$ & Average & RMSD \\
\hline Scale factor, SF & 0,0214 & 0,021 & 0,0116 & 0,0121 & 0,0128 & 0,0129 & 0 & 0,0033 \\
\hline $\begin{array}{c}\text { Mismatch, } \Delta \varphi \\
\text { [g/deg/s] }\end{array}$ & $-0,003$ & $-0,0029$ & $-0,0025$ & $-0,0023$ & $-0,003$ & $-0,003$ & $-0,001$ & 0,001 \\
\hline $\begin{array}{c}\text { Non- } \\
\text { orthogonality of } \\
\text { axes, } \mathbf{N}\end{array}$ & 0,0612 & 0,0673 & 0,051 & 0,0514 & $-0,057$ & $-0,055$ & 0,05 & 0,05 \\
\hline
\end{tabular}

The effectiveness of the proposed calibration method can be proved by graphs of the difference in calibration results achieved with the MMPM and the method proposed in this article (Figure 3). 


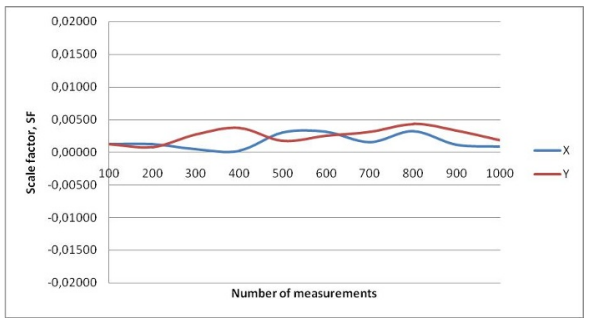

(a)

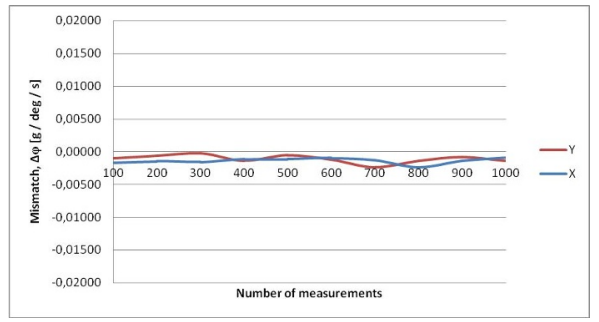

(b)

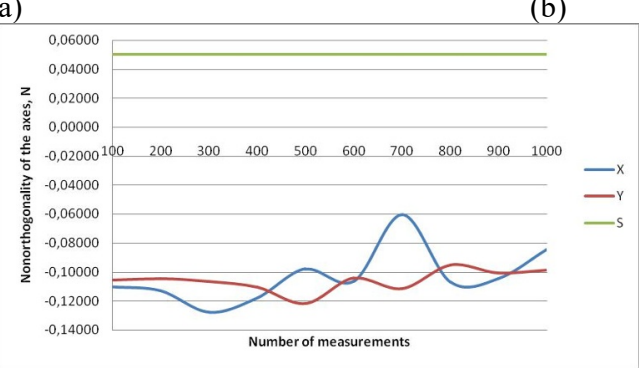

(c)

Fig. 3. Graphs of the difference in calibration results achieved with the MMPM and the proposed method. (a) Scale factor, SF; (b) Mismatch, $\Delta \varphi$; (c) Non-orthogonality of the axes, N.

In Figures $3 \mathrm{a}-3 \mathrm{c}, \mathrm{X}$ is the difference between the real measurement values with respect to the calibrated by the MMPM method, and $\mathrm{Y}$ is the difference between the real measurement values and the calibrated methods proposed in the paper. As can be seen in the figures, the proposed method has better error compensation characteristics.

\section{Acknowledgments}

The work was carried out as part of the research on the project, support by the Ministry of Education and Science of the Russian Federation No. 5.3606.2017/PCH

\section{References}

1. Andrejašic M 2008 MEMS accelerometers (Marec, University of Ljubljana) pp 1-17

2. Mohn-Yasin F, Korman C E and Nagel D J 2003 Solid-State Electronics 47 357-60

3. Beeby S, Ensell G and Kraft M 2004 MEMS mechanical sensors (Artech house inc., USA) P 282

4. Mukherjee R; Basu J; Mandal P and Prasanta K G 2017 J. of Micromechanics and Microengineering 27 1-34

5. Doscher J 1998 Accelerometer Design and Applications (Analog Devices)

6. Grecheneva A, Konstantinov I and Kuzichkin O 2016 SGEM2016 Conf. Proc. 28 June-6 Jule Albena vol. 1 (Albena), pp 829-36

7. Kuzichkin O R, Grecheneva A V, Mikhaleva E S and Dorofeev N V 2017 J. of Fundamental and Appl. Sci. 9 1871-84

8. Grecheneva A, Kuzichkin O and Dorofeev N 2016 SGEM2016 Conf. Proc. 28 June6 Jule Albena vol. 1 (Albena), pp 383-90

9. Grecheneva A, Kuzichkin O and Dorofeev N 2017 Int. Conf. on Industrial Engineering, Applications and Manufacturing 16-19 May St. Peterburg (St. Peterburg: IEEE) P 4 
10. Shavrin V V, Konakov A S and Tislenko V I 2012 Reports of TUSUR 2 265-269

11. Specification for the inertial measuring unit ADIS16354 High Precision Tri-Axis Inertial Sensor http://www.alldatasheet.com/datasheetpdf/pdf/224613/AD/ADIS16354.html 